\title{
An Improved Method for Moving Target Detection Based on Spatial-temporal Fusion Filtering
}

\author{
Zhongmin Li, Haochen Wu and Guowei Zou \\ School of Information Engineering \\ Nanchang Hangkong University \\ Nanchang, China \\ zhongmli@163.com, 464417131@qq.com, 824206587@qq.com
}

\begin{abstract}
Infrared weak small target detection is one of the key technologies in the infrared early warning system, infrared imaging guidance system and wide-field view surveillance system etc. In the complex and low signal-to-noise ratio background environment, the target has only a few pixels. There is no shape and texture information to use. All of the above issues bring great difficulties to infrared dim-small target detection. In this paper, in order to deal with the shortcoming of large pixel tracking in moving target detection algorithm based on time domain filtering, apply the dim target detection based on Laplace operator to the subject of this paper, the false alarm points obviously to be less, and the target point is usually in the form of a single pixel, lay a good foundation for reducing the complexity of the algorithm.
\end{abstract}

Keywords-moving target detection; spatial-temporal fusion filtering; Laplace operator

\section{INTRODUCTION}

With the rapid development of modern military technology, all kinds of precision guided missiles is playing an important role in modern warfare, it can precision strike with the military targets on the ground, planes, ships and aircraft carrier battle platform and so on. With the corresponding, in order to ensure the safety of the operation platform, we need to effective detection, tracking and confrontation for the hit target. Early target detection and tracking system mainly uses radar technology; it has advantages with all-weather and effect of distance. However, due to the inherent external radiation characteristics of radar, it becomes easy to expose and suffer from the anti-radiation missile attacks. However infrared detector passive receives the target radiation heat to detect the target, and itself does not radiate energy. Compare with the radar, it has the advantages of good concealment, strong ability to resist electromagnetic interference.

Moreover, with the rapid development of modern aerospace technology, the flight speed, maneuverability and sensitivity of the precision-guided missiles is becomes higher and higher. such as the $\mathrm{X}-51$ of America , the Kh-15C of Russia and the ASM - 3 of Japan , etc. their speed of cruise missile to be 5 -7 Mach , and the maneuverability is very strong, so it put higher requirements forward on the performance of target detection and tracking system. In order to strive for long enough reaction

This work was financially supported by the Programs of the National Natural Science Foundation of China (Grant No. 61263040). time, this must be detection and tracking the target at a distance. However, remote imaging is highly affected by the background, the SNR of the image that received by infrared sensors is low. And the pixels of the target that in the image is very little, it causes the target detection and tracking became very difficult.

Infrared small target detection method is mainly divided into two kinds: one is the detect before track method and the other is the track before detect method.

The detect before track firstly according to the short gray features of single frame image to detect the possible target points, then according to short-term movement speed characteristic of the target to eliminate the false target and obtain the real target trajectory, the most typical weak target detection algorithm is based on all kinds of single frame detection and multiple frames trajectory analysis. because the global characteristics of weak small targets is not very obvious, it generally only for value point of local extreme, due to the weak target detection algorithm which based on global features mostly have a poor performance, we generally choose target detection algorithm based on local feature. Fengyun Zhu et al put forward a kind of motor weak target detection algorithm base on the grayscale reverse phase feature of neighborhood [1]; Wang and others by using small surface model to fit the neighborhood surface of image, it base on the extreme conditions to obtain the candidate target [2]; Songtao Liu presented fingerprint image segmentation method based on cellular automaton [3]. in addition, There are others detection algorithm of single frame weak target which based on genetic algorithm [4], neural network[5] and local entropy of image [6], etc. after winning point set of the candidate target, according to movement characteristics of the target, we could eliminate the false target of the image sequences and get its true trajectory. Diana presented target detection algorithm based on pipeline filter [7]. There are also some algorithms using likelihood ratio [8], neural network algorithm, the three dimensional spatial filter and two-dimensional matched filter [9] etc. they extracted the real goal from candidate target.

The track before detect algorithm is to search all possible trajectories of the target, and complete accumulate energy of the target, and then get the posteriori probability of each trajectory, finally using the threshold value to judge the real target motion trajectory. Reed put forward a three-dimensional matched filtering algorithm to improve signal-to-noise ratio of 
the image [10]; [11] put forward a kind of track before detection algorithm based on Multistage hypothesis testing; Bin Wu and others used improved power law detector into the small moving target detection which in infrared image sequences of strong background. In addition, there are some track before detection algorithm based on genetic algorithm [12], neural network, image matching method and the wavelet multi-scale analysis [13] .track before detection algorithm need to track all possible trajectory of the whole space-time domain, its algorithm computational is complexity and storage capacity is very big, it has long testing time and easily lose synchronization with the actual moving target [14], its realtime implementation is difficult, it generally used to establish relatively a complete model and processing method, it used [15] in the practical engineering application less.

In addition, there is a detection algorithm based on machine learning, it put the target detection problem transform into a pattern classification problem. Then according different learning algorithms to train the target model and the background model, and using the target model and the background model to classify the input image. that is said to extract sub images of the input image in turn, then according the discriminate rules to determine whether the image block contains the target or not, among them, the typical method have principal component analysis method, the probabilistic principal component analysis method and infrared small target detection algorithm based on image sparse representation [16]. At present, this kind of algorithm is still in the phase of theoretical research.

In this paper, in order to deal with the shortcoming of large pixel tracking in moving target detection algorithm based on time domain filtering, we present an improved method for moving target detection based on spatial-temporal fusion filtering.

\section{BASIC IDEA}

Since the target detection method based on spatial-temporal filtering with a large pixel tracking shortcoming, it is difficult to realize real-time detection of small targets. Search for a less pixel tracking target detection method with simple algorithm becomes meaningful. In this paper, the target detection method based on Laplace operator, applied to the small target detection in infrared sequence image under cloud background, obtained the very good effect. And on the basis of this method, improved the moving target detection method based on interframe spatial correlation of the moving target, further reducing the complexity of the algorithm. Finally, based on the temporal correlation method, put forward an improved spatial-temporal fusion filtering method for moving target detection.

\section{EXPERIMENTAL RESULTS}

We got the detection result of point target detection based on Laplace operator, and then detect the result through moving target spatial correlation detection, as shown in Fig. 1. Firstly, find the coordinates of 4 candidate points in the image, store them in the array $\mathrm{P}$ and mark with a green "+” , as shown in Fig. 1. Then traverse their 8-neighbor in the second frame to search eligible point, if we find a point in the 8-neighbor of one candidate points that its time domain signal correlates with the candidate point, then we can judge the candidate point is the target point, mark it with a small red circle, otherwise, if not find a eligible point, the candidate point is false alarm point or random noise. By the method mentioned in the paper, we have found the target point in first frame successfully; its coordinates are $(152,137)$, as shown in Fig. 1(d). Loop the process above, found the target points in second and third frame, their coordinates are $(152,142)$ and $(150,147)$, as shown in Fig. 1(e) and Fig. 1(f).

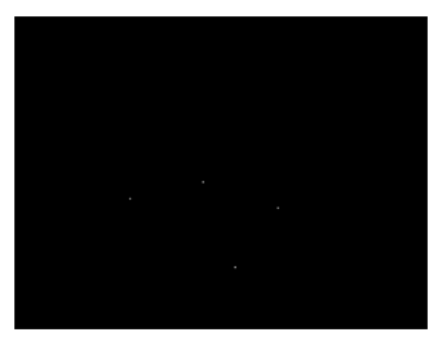

(a)

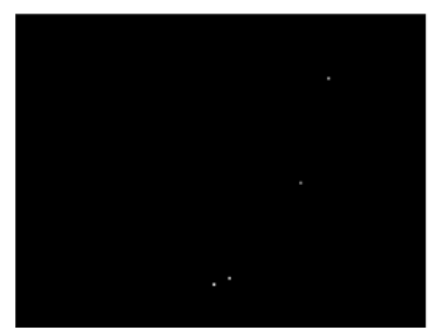

(c)

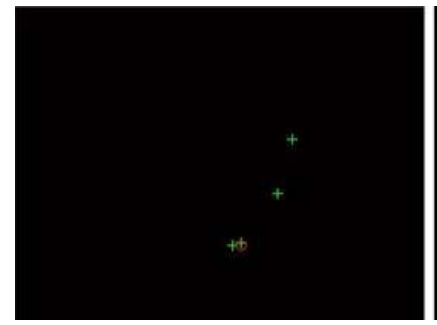

(e)

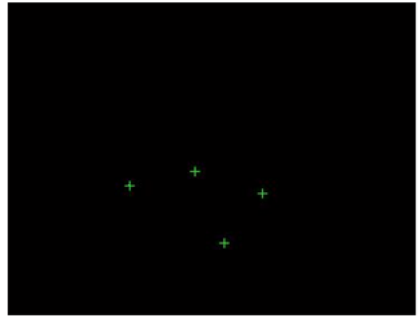

(b)

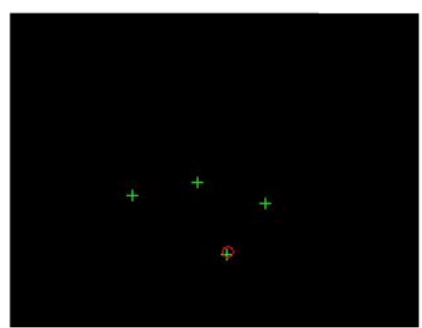

(d)

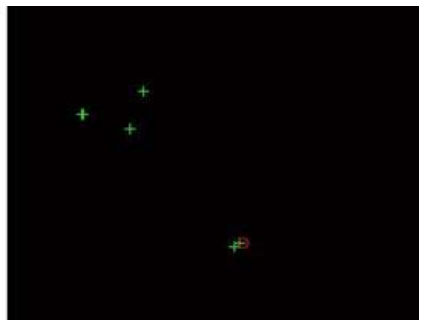

(f)
Fig. 1. Detection results: (a)first frame image after spatial filtering (b)first frame marked with candidate points (c)second frame image after spatial filtering (d) first frame image marked with target point (e) second frame image marked with target point (f)third frame image marked with target point.

\section{SUMMARY}

In this paper, in order to deal with the shortcoming of large pixel tracking in moving target detection algorithm based on time domain filtering, apply the dim target detection based on Laplace operator to the subject of this paper, the false alarm points obviously to be less, and the target point is usually in the form of a single pixel, lay a good foundation for reducing the complexity of the algorithm. Then on the basis of this method, improved the moving target detection method based on interframe spatial correlation of the moving target, which can effectively reduce the complexity of the original algorithm. Finally, based on the temporal correlation method, put forward 
an improved spatial-temporal fusion filtering method for moving dim target detection.

\section{ACKNOWLEDGMENT}

This work was financially supported by the Programs of the National Natural Science Foundation of China (Grant No. 61263040).

\section{REFERENCES}

[1] F. Zhu, S. Qin, “A detection alogrithm for maneuvering IR point target and its performance evaluation,” International Coference on Machatronic and Embedded System and Application, pp. 1-5, 2006.

[2] G. D. Wang, Ch. Y. Chen and X. B. Shen, "Facet based on infrared small target detection method,” Electronics Letters, vol. 41, no. 22, pp. 885-888, 2005.

[3] S. Liu and S. Yang, "Infrared dim-small target image segmentation based on cellular automata,” Journal of infrared and millimeter wave, vol. 27, no. 1, pp. 42-46, 2008.

[4] L. Cao, "An unsupervised target detection algorithm in SAR images," Conference on Synthetic Aperture Radar, pp. 529-532, 2007.

[5] H. Leung, N. Dubash, and N.Xie, "Detection of small objects in clutter using a Ga-RBF neural network," IEEE Trans. on Aerospace and Electronic Systems, vol. 38, no. 1, pp. 98-118, 2002.

[6] G. Wang, J. Tian, and J. Liu, "Infrared small target image detection based on local entropy,” Infrared and laser engineering, vol. 29, no. 4, pp. 26-29, 2000.
[7] M. Diani, G. Corsini, and A. Baldacci, "Space-time processing for the detection of airborne targets in IR image sequences,” IEE Processing on Vision, Image Signal Processing, vol. 148, no. 3, pp. 151-157, 2001.

[8] J. F. Y. Cheung, M. C. Wicks, G. J. Genello, et al., "Statistical theory for optimal detection of moving objects in variable corruptive noise," IEEE Trans. on Image Processing, vol. 8, no.12, pp. 1772-1787, 1999.

[9] S. A. Nichols and R. B. Naylor, "Reliable motion detection of small targets in video with low signal-to-clutter ratios,” SPIE, vol. 2561, pp. 168-177, 1995.

[10] I. S. Reed, R. M. Gagliardi, and L. Stotts, "Optical moving target detection with 3-D matches filtering,” IEEE Trans on Aeros. Electron. Sys., vol. 24, no. 4, pp. 327-336, 1998.

[11] S. D. Blostein and H. S. Richardson, “A sequential detection approach to target tracking,” IEEE Trans on AES, vol. 30, no. 1, pp. 197-211, 1994.

[12] B. Wu and H. Ji, "Improved power-law-detector-based moving small dim target detection in infrared images,” Optical Engeering, vol. 47, no. 1, pp. 010503, 2008.

[13] D. J. C. Andrew, et al., "Inexact graph matching using genetic search,” Pattern Recognition, vol. 30, no. 7, pp. 953-970, 1997.

[14] M. Irani, P. Anandan, "A unified approach to moving object detection in 2D and 3D scenes," IEEE Trans on Pattern Analysis and Machine Intelligence, vol. 20, no. 6, pp. 577-589, 1998.

[15] L. Yang, "Small target detection and tracking algorithm research under the condition of complex background,” Wuhan: Huazhong University of science and technology doctoral thesis, 2006.

[16] J. Zhao, Z. Tang, jie Yang, et al., "The infrared small target detection algorithm based on image sparse representation,” Journal of infrared and millimeter wave, vol. 30, no. 2, pp. 156-161, 166, 2011. 